\title{
Efficient Expression of Exogenous Genes in Primary Vascular Cells Using IRES-Based Retroviral Vectors
}

BioTechniques 32:830-843 (April 2002)

\author{
Kyle J. Garton, Nicola Ferri, \\ and Elaine W. Raines \\ University of Washington \\ School of Medicine, Seattle, \\ WA, USA
}

\footnotetext{
ABSTRACT

Analysis of gene function in primary vascular cells has been particularly limited by low transfection efficiencies. Using internal ribosomal entry site (IRES)-based retroviral vectors, we demonstrate efficient infection (range of 45\%-95\%) of primary human endothelial and smooth muscle cells with genes varying in size from 1.3 to $4.5 \mathrm{~kb}$. Because IRES vectors are designed to allow the expression of two genes from a single $m R N A$, we can show excellent correlation between the expression of a reporter gene and an inserted gene of interest. Reporter gene expression allows rapid (24-48 h) and unambiguous identification of transduced cells. Additionally, reporter gene expression can be used to isolate subpopulations of cells that express distinct levels of cistron 1 genes by flow cytometry, and sorted cells maintain relative levels of gene expression over multiple passages in culture. Two examples of the usefulness of these vectors to characterize gene function in primary vascular cells include (i) the inhibition of endothelial cell inflammatory responses in a polyclonal population by the expression of a dominant negative inhibitor of nuclear factor- $\kappa B$ and (ii) monitoring the in vitro evolution of smooth muscle cells provided with a selective growth advantage by transduction with telomerase. Potential applications of retroviral expression strategies in vascular biology are also discussed.
}

\section{INTRODUCTION}

The characterization of gene function in vascular cells, including endothelial and smooth muscle cells (SMCs), has been limited by two major factors. First, suitable cell lines that maintain phenotypic and functional characteristics of primary vascular cells are lacking. Second, expression of exogenous genes has been difficult because of the extremely low transfection efficiency of primary vascular cells. As a result, many studies have been performed in cell lines of uncertain physiologic relevance and/or in primary vascular cells transduced at very low efficiencies.

An ideal strategy for gene expression in mammalian cells should meet several important criteria. First, stable and efficient transduction of primary, non-transformed cells should be possible to avoid the use of cell lines. The method of gene transduction should be nontoxic, and appropriate controls for expression of selectable markers should be used. Further, gene transduction and expression or selection should be rapid for use in primary cells with a limited in vitro replicative capacity. Additionally, transduced cells should be easily identified and, if necessary, isolated from polyclonal cell mixtures. Ideally, it should also be possible to vary gene expression levels to characterize gene dosage effects. Finally, analysis of gene function(s) should be possible within large polyclonal populations of transduced cells to avoid potential clonal artifacts.

Recent advances in expression techniques utilizing single-transcript retroviral vectors allow many of these criteria to be fulfilled for the first time using primary vascular cells. A limited number of recent studies have shown that single-transcript retroviral constructs can be used to efficiently transduce murine endothelial and canine vascular SMCs (12,15). However, systematic characterizations of the utility of these constructs in primary human vascular cells are not available. Here we demonstrate the utility of single-transcript retroviral vectors for the study of gene function in primary human endothelial cells and SMCs.

\section{MATERIALS AND METHODS}

\section{Cell Culture and Reagents}

Human umbilical vein endothelial cells (HUVECs) were isolated and cultured in RPMI 1640, supplemented with $15 \%$ FCS, $0.4 \%$ bovine pituitary brain extract (Invitrogen, Carlsbad, CA, USA), and $50 \mu \mathrm{g} / \mathrm{mL}$ heparin as previously described (4). Human arterial SMCs were derived from the media of normal thoracic aortas by explant and were maintained in DMEM supplemented with $10 \%$ FCS. We used the following antibodies: anti-HA (clone HA.11; Babco, Richmond, CA, USA), anti-E-selectin (Clone 68-5H11; BD Pharmingen, San Diego, CA, USA), phycoerythrin (PE)-labeled anti-ICAM1 (clone HA58; BD Pharmingen), and PE-labeled goat anti-mouse IgG (Biomeda, Foster City, CA, USA).

\section{Protein Analysis}

For immunoblotting, cells were lysed in $50 \mathrm{mM}$ Tris-HCl, pH 7.4, 250 
$\mathrm{mM} \mathrm{NaCl}, 0.5 \%$ Nonidet ${ }^{\circledR} \mathrm{P}-40,10 \%$ glycerol, $5 \mathrm{mM}$ EDTA, $50 \mathrm{mM} \mathrm{NaF}$, $0.5 \mathrm{mM} \mathrm{Na} \mathrm{VO}_{4}, 10 \mathrm{mM} \beta$-glycerophosphate, PMSF, $5 \mu \mathrm{g} / \mathrm{mL}$ leupeptin and aprotinin. Lysates were separated on $10 \%$ or $15 \%$ SDS-PAGE, and proteins were transferred to Immobilon ${ }^{\mathrm{TM}}$ membranes (Millipore, Bedford, MA, USA) and immunoblotted with specific antibodies. Immunoblots were visualized by enhanced chemiluminescence (Amersham Biosciences, Piscataway, NJ, USA).

\section{Generation of Retroviral Vectors, Retrovirus Production, and Infec- tion of Endothelial Cells and SMCs}

All retroviral expression plasmids were constructed using the $\mathrm{pBMN}$ IRES-EGFP, pBMN-IRES-Lyt2a, and pBMN-IRES-PURO retroviral vectors and standard molecular biology techniques $(8,9)$. The $\mathrm{pBMN}$-IRES retroviral vectors contain a single-transcript expression cassette in which a retroviral promoter drives the expression of two genes. The pBMN-IRES-EGFP vector utilizes enhanced GFP (EGFP) as a second cistron reporter gene, pBMN-IRESLyt2a, a splice variant of murine CD8, and $\mathrm{pBMN}$-IRES-PURO, the puromycin resistance gene. The cDNAs of human pro-HB-EGF, human MDC15, and human telomerase (hTERT) were cloned into the BamHI/NotI sites of pBMN-IRES-EGFP and I $\kappa B \alpha-$ Ser into pBMN-IRES-Lyt2a. EGFP was cloned into the BamHI/NotI sites of pBMNIRES-PURO.

Amphotrophic retrovirus was prepared with the use of Phoenix-A packaging cells as previously described (9). After transfection of Phoenix-A packaging cells, the medium was changed at $10 \mathrm{~h}$ and again at $24 \mathrm{~h}$ after transfection. Virus collected between 24 and 48 $\mathrm{h}$ after transfection was used for infection. Retroviral titers between $10^{6}$ and $2 \times 10^{7} \mathrm{cfu} / \mathrm{mL}$ were determined by limiting dilution with NIH3T3 cells. For infection, $4 \times 10^{5}$ HUVECs or SMCs were plated in $25-\mathrm{cm}^{2}$ flasks 24 $\mathrm{h}$ before infection in normal growth medium to obtain exponentially growing cultures. The medium was replaced with $4 \mathrm{~mL}$ retroviral supernatant (approximate MOI $2.5-25 \mathrm{cfu} / \mathrm{cell}$ ) supplemented with $4 \mu \mathrm{g} / \mathrm{mL}$ polybrene.
After $12 \mathrm{~h}$, retroviral supernatants were removed and replaced with fresh normal growth medium for $48 \mathrm{~h}$. In these conditions, no apparent toxicity was observed after a 12-h exposure to retroviral supernatants containing polybrene in either HUVECs or SMCs.

\section{Flow Cytometry}

For the analysis of EGFP expression after retroviral infection, cells were harvested by brief trypsinization, washed in PBS, and fixed with $2 \%$ paraformaldehyde. The percentage of EGFP $(+)$ cells was determined by flow cytometry by the use of a FACScan ${ }^{\mathrm{TM}}$ flow cytometer and analyzed with $\mathrm{BD}$ CellQuest $^{\mathrm{TM}}$ (both from BD Biosciences, San Jose, CA, USA). For two-color analysis of cells expressing the CD8 (Lyt2) retroviral cell surface marker and an EGFP reporter gene, cells were stained with a PE-conjugated anti-CD8 antibody (BD Pharmingen) for $30 \mathrm{~min}$ on ice, followed by three washes in PBS $+0.25 \%$ BSA and post-fixed with $2 \%$ paraformaldehyde before analysis. Similarly, expression of adhesion molecules (E-selectin and ICAM-1) or hemagglutinin (HA)-tagged HB-EGF was determined by staining with biotinylated primary antibodies followed by PE-conjugated second antibodies. Cell-surface protein expression was quantified as the mean fluorescence intensity of greater than 10000 gated cells.

\section{RESULTS AND DISCUSSION}

Single-transcript expression cassettes (STCs) are designed to allow the expression of two genes from a single mRNA. STCs contain a single promoter and two cistrons separated by an internal ribosomal entry site (IRES) sequence that allows eukaryotic ribosomes to initiate $5^{\prime}$-cap-independent translation of a second cistron gene (7, 13) (Figure 1A). Typical cassettes contain a gene of interest cloned into the first cistron and a selection or reporter gene, such as the EGFP, cloned into the second cistron. A major advantage of STCs is that expression of the second cistron reporter gene typically ensures expression of the first cistron gene. To determine the usefulness of retroviral vectors for introducing genes into HUVECs and human aortic SMCs, we infected these cells with pBM-IRESEGFP retrovirus $(8,9)$. In the absence of a first cistron gene, HUVECs and SMCs were infected at an infection efficiency of $93 \%$ and $75 \%$, respectively, as determined by flow cytometric analysis of EGFP expression (Figure 1B). Similar results are seen using a specific antibody against a truncated cell-surface murine CD8 reporter gene in IRESCD8-infected cells (not shown).

IRES-based retroviral vectors provide an effective strategy for delivering genes of varying sizes to primary vascular cells at high efficiency. We find that inclusion of a gene into the first cistron generally leads to decreased infection efficiencies with an inverse relationship between the size of the cDNA and the efficiency of retroviral infection (Figure 1B). However, infection efficiencies with a large $4.5-\mathrm{kb}$ first cistron cDNA (hTERT) were still 56\% and $45 \%$ for HUVECs and SMCs, respectively. Retroviral infection can also be used to simultaneously express two genes in primary vascular cells. Sequential infection of HUVECs with IRES-EGFP retrovirus followed by an IRES-CD8 retrovirus yields approximately 63\% EGFP(+)CD8(+) cells by flow cytometry (Figure 1C). Alternatively, simultaneous co-infection with a 50:50 mixture of IRES-EGFP and IRES-CD8 retrovirus yields approximately 40\% EGFP $(+) \mathrm{CD} 8(+)$ cells (Figure 1C). Sequential and simultaneous infection of SMCs is also possible and yields approximately $20 \%$ and $11 \% \operatorname{EGFP}(+) \operatorname{CD} 8(+)$ cells, respectively (Figure 1C). This approach is particularly useful in characterizing gene-gene interactions, such as the effect of overexpression of a wild-type versus a dominant negative mutant within a single polyclonal population of primary vascular cells.

Stable integration of IRES-based retroviral cassettes into the host cell genome allows for the isolation of purified populations of infected cells expressing different levels of reporter genes (therefore, cistron 1 genes) by flow cytometry. The metalloproteinasedisintegrin protein 15 (MDC15) is a 120-kDa cell-surface protein, encoded by a $2.5-\mathrm{kb}$ cDNA, which is expressed by endothelial cells and SMCs in vitro 
and up-regulated within lesions of atherosclerosis in vivo (4). We asked whether EGFP reporter gene expression could be used to isolate distinct populations of MDC15-expressing cells and if gene expression is maintained over multiple passages in culture. Human SMCs were infected with MDC15(HA)-IRES-EGFP virus encoding an HA epitope-tagged version of human
MDC15 or control IRES-EGFP virus (Figure 2A). Polyclonal populations of cells $\left(3 \times 10^{5}\right.$ each) expressing low or high levels of the EGFP reporter gene were then isolated by flow cytometry. Immediate post-sort analysis confirmed the isolation of distinct populations of cells based on EGFP expression (Figure 2A). Cells were subcultured for an additional passage and analyzed for expres- sion of the EGFP reporter gene by flow cytometry (data not shown) and for expression of HA-tagged MDC15 by Western blot analysis (Figure 2A). Sorted human SMCs maintained their relative expression levels of EGFP, and there was excellent correlation between EGFP and MDC15 expression.

To more rigorously test whether EGFP reporter gene and relative

A

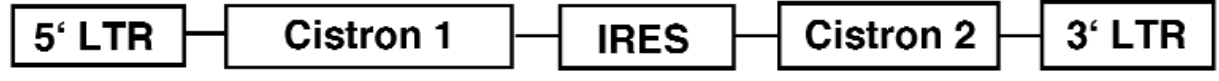

B

HUVECS
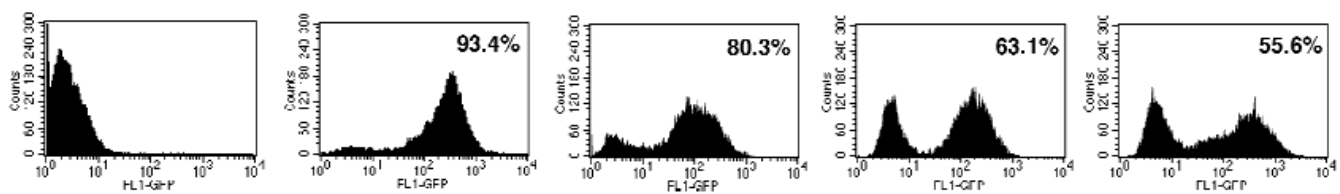

SMCs
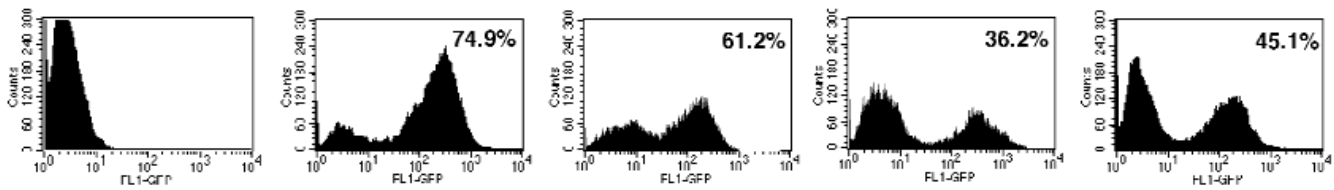

Cistron 1

cDNA Size: Uninfected

$0 \mathrm{kB}$

$1.3 \mathrm{kB}$

$2.5 \mathrm{kB}$

$4.5 \mathrm{kB}$

C

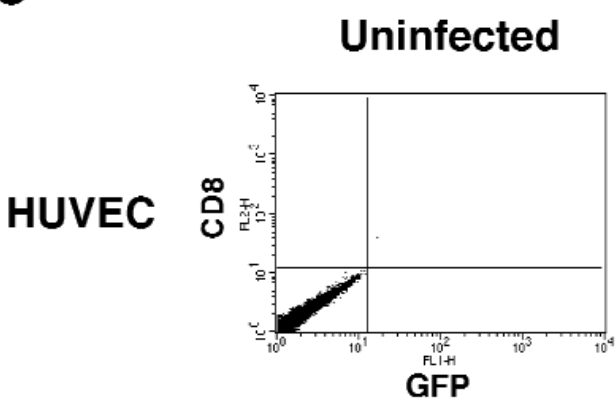

Sequential
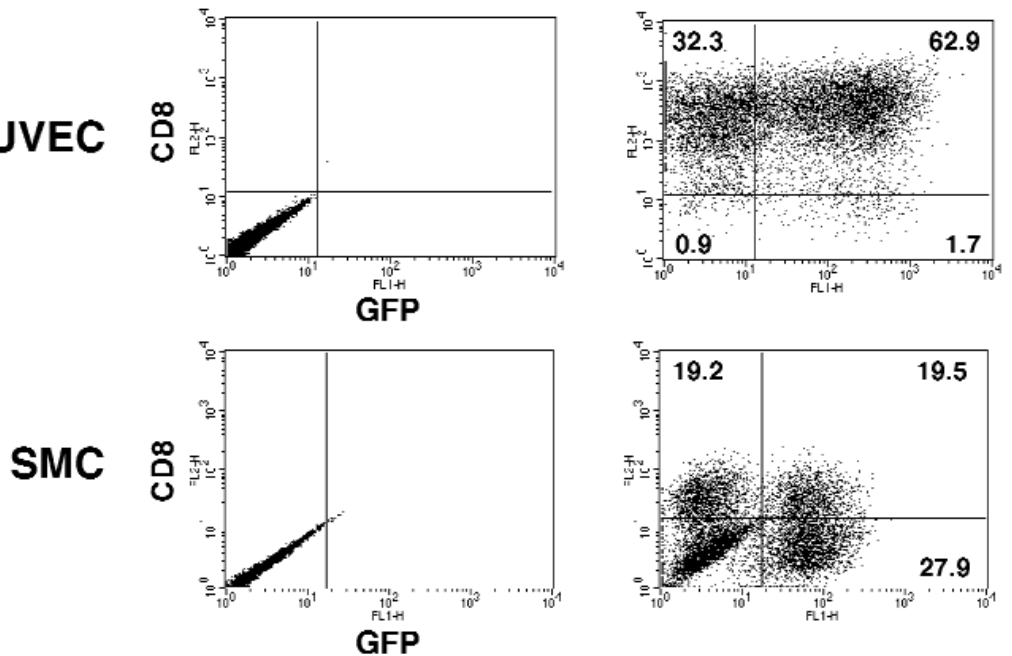

Simultaneous
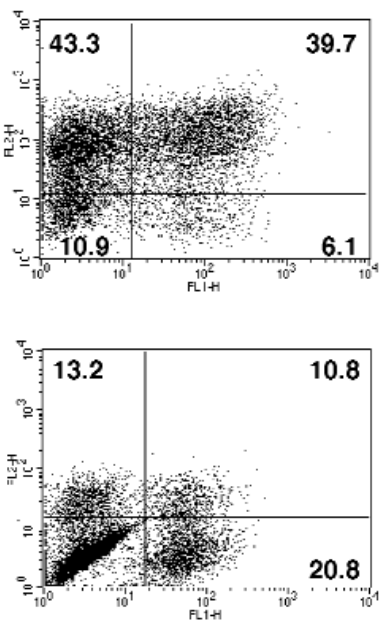

Figure 1. IRES-based retroviral vectors provide an effective strategy for delivering genes of varying sizes to primary cells at high efficiency. (A) General structure of STCs. Transcription is initiated by promoter sequences within the viral $5^{\prime}$ long terminal repeat (LTR) and terminated by polyadenylation sequences within the $3^{\prime}$ LTR. Translation of cistrons 1 and 2 from a single mRNA proceeds by ribosome binding to $5^{\prime}$ Cap and IRES sequences, respectively. (B) Retroviral infection efficiency of HUVECs and SMCs with retroviral cassettes containing varying sized cistron $1 \mathrm{cDNAs}(0$-kb no cistron $1 \mathrm{cDNA}, 1.3$-kb human pro-HB-EGF, 2.5-kb human MDC15, 4.5-kb hTERT). Retroviral infection efficiencies were determined by flow cytometry. (C) Sequential versus simultaneous co-infection of HUVECs and SMCs. For sequential infection, cells were infected at passage 2 with IRES-EGFP retrovirus and at passage 3 with IRESCD8 virus. Simultaneous infection was achieved with a 50:50 mixture of IRES-EGFP and IRES-CD8 virus at passage 2. SMCs were infected with an identical protocol at passages 7 and 8 . The percentage of $\operatorname{EGFP(+),~CD8(+),~or~EGFP(+)CD8(+)~cells~in~each~population~is~indicated.~}$ 
A

A IRES-EGFP MDC-IRES-EgFP
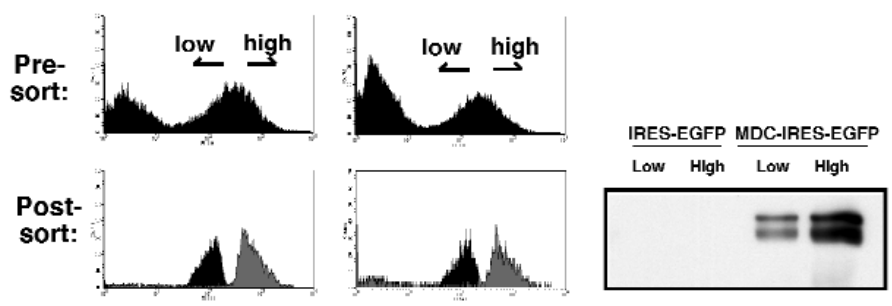

B IRES-EGFP

Pre-
sort:
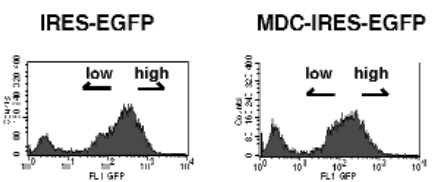

Postsort:
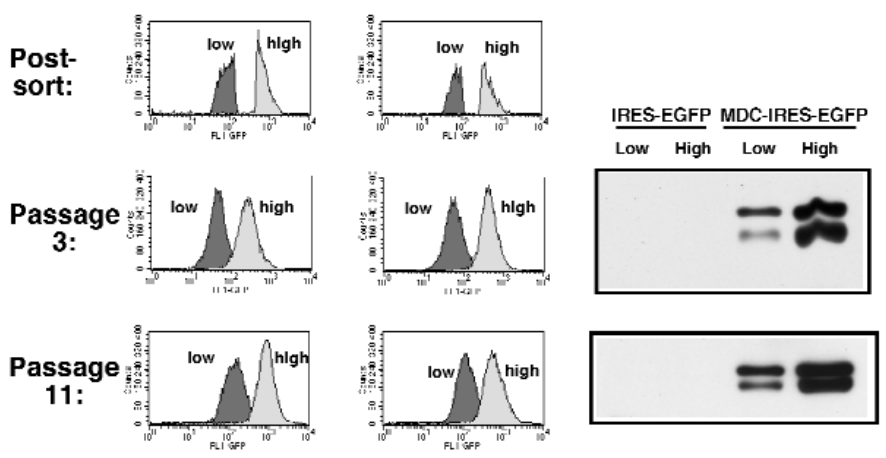

C
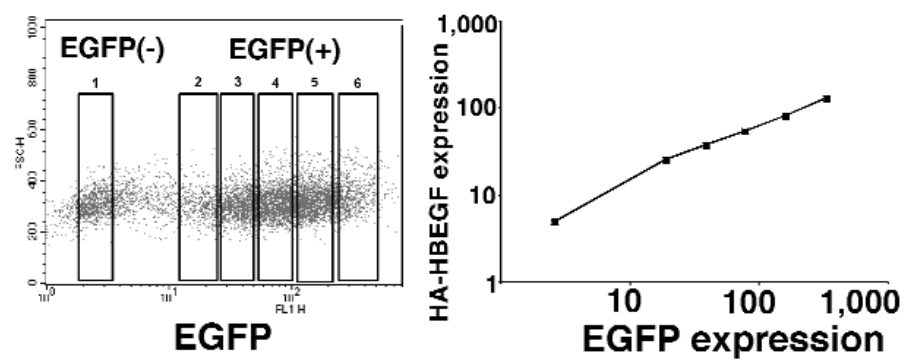

D

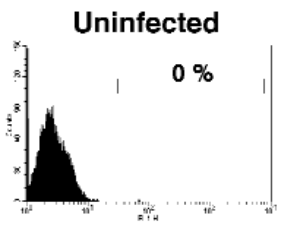

EGFP-IRES-PURO
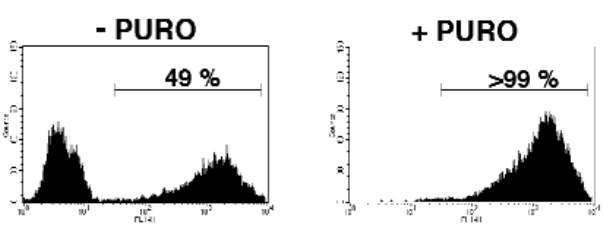

MDC15 expression is stable over multiple passages in culture, cell sorting experiments were repeated using NIH3T3 cells that are a uniform replicative cell population capable of extended population doublings. Low and high expressing populations of NIH3T3 cells were subsequently subcultured for 3 or 11 passages (approximately 20 and 75 population doublings, respectively) (Figure 2B). The sorted NIH3T3 cells maintained their different expression levels as determined by flow cytometry and Western blot analysis (Figure 2B). These results demonstrate that EGFP reporter gene expression can be used to isolate distinct populations of cells expressing different levels of first cistron genes by flow cytometry and that stable levels of gene expression are maintained over multiple passages in vitro.

We next wanted to characterize further the correlation between expression of a reporter gene and a transmembrane protein cloned into the first cistron of an IRES-based retroviral vector in primary vascular cells. Pro-heparin-binding epidermal growth factor (Pro-HBEGF) is a cell-surface growth factor that plays an important role in the migration and proliferation of vascular cells (14). It has been shown previously

Figure 2. Stable integration of IRES-based retroviral cassettes into the host cell genome allows isolation and analysis of populations of cells expressing different levels of cistron 1 genes. (A) Human aortic SMCs were infected with control IRES-EGFP or MDC15(HA)-IRESEGFP retrovirus. Cell sorting was used to isolate $3 \times 10^{5}$ cells expressing low and high levels of the EGFP reporter gene from each population, which was verified by post-sort flow cytometric analysis. Western blot analysis was used to determine the expression of HA-tagged MDC15 in isolated populations. (B) NIH3T3 cells infected as in part A were sorted, and populations were subcultured for 3 or 11 passages and analyzed for EGFP and HA-MDC15 expression as in part A. (C) Analysis was made of EGFP and HA-tagged HB-EGF expression by flow cytometry in HUVECs infected with HA-HB-EGF-IRES-EGFP retrovirus. HA-HB-EGF expression was determined in cells expressing increasing levels of the EGFP reporter gene (gates 2-6, left) and in control uninfected cells (gate 1) and plotted as a function of EGFP expression (right). (D) HUVECs were infected with pBM-EGFP-IRESPURO retrovirus at a titer to achieve an approximately $50 \%$ infection efficiency. The resulting cells were split into two populations and grown in the presence or absence of puromycin $(2.5$ $\mu \mathrm{g} / \mathrm{mL}$ ) for $24 \mathrm{~h}$. EGFP expression was determined by flow cytometry. 
that cell-surface levels of pro-HB-EGF can be monitored by analysis of a version of pro-HB-EGF containing an HA epitope tag at the $\mathrm{N}$-terminus of the extracellular domain (3). HA-pro-HBEGF was cloned into the IRES-EGFP retroviral vector and subsequently used for the infection of HUVECs, leading to the generation of distinct $\operatorname{EGFP(-)}$ and $\mathrm{EGFP}(+)$ populations by flow cytometry (Figure 2C). Coordinately, HA-HB-EGF expression was determined by the use of an antibody against the HA-epitope tag and a PE-conjugated secondary antibody. The correlation between EGFP and HA-HB-EGF expression was determined by gating on populations of cells expressing increasing levels of the EGFP reporter gene, followed by the measurement of the relative HB-EGF expression (PE mean fluorescence intensity) (Figure 2C). These results demonstrate an excellent correlation between the expression of the first cistron HA-HB-EGF and the second cistron EGFP reporter gene (Figure 2C). Moreover, the data show that expression levels achieved by retroviral transduction do not saturate the intracellular machinery responsible for the processing and cell-surface targeting of HB-EGF.

Previous studies have shown that endothelial cells can be isolated by flow cytometry and subsequently used for functional analysis (10) after co-transfection with a plasmid expressing GFP and a second plasmid expressing a gene of interest. A major limitation of this approach is that expression of the GFP reporter and the gene of interest are not linked and are transient. In addition, although primary vascular cells can be sorted and subsequently subcultured (Figure 2A and data not shown), cell sorting is expensive, time consuming, and not readily available to many laboratories. Therefore, we generated an IRES-based retroviral vector expressing the puromycin resistance gene as a selectable second cistron gene (pBMIRES-PURO) and tested the utility of this construct in generating highly purified populations of retrovirally infected primary human endothelial cells. Puromycin selection was chosen because puromycin very rapidly kills cells (1-2 days) and is inexpensive (6). A test construct was also generated in which
EGFP was cloned into the pBM-IRESPURO vector as a first cistron gene and subsequently used to infect HUVECs at approximately 50\% efficiency (Figure 2D). Infected cells were subsequently split into two populations and grown in the presence or absence of puromycin $(2.5 \mu \mathrm{g} / \mathrm{mL})$ for $24 \mathrm{~h}$, and EGFP expression was determined by flow cytometry. Cells grown in the absence of puromycin remained approximately $50 \% \operatorname{EGFP}(+)$, whereas those in the presence of puromycin were essentially $100 \% \operatorname{EGFP}(+)$ after $24 \mathrm{~h}$ of selection (Figure 2D). An identical result is obtained with an overnight $(12 \mathrm{~h})$ puromycin selection (data not shown). Using this approach, we can obtain virtually $100 \%$ infected populations of primary endothelial cells within $24 \mathrm{~h}$ with

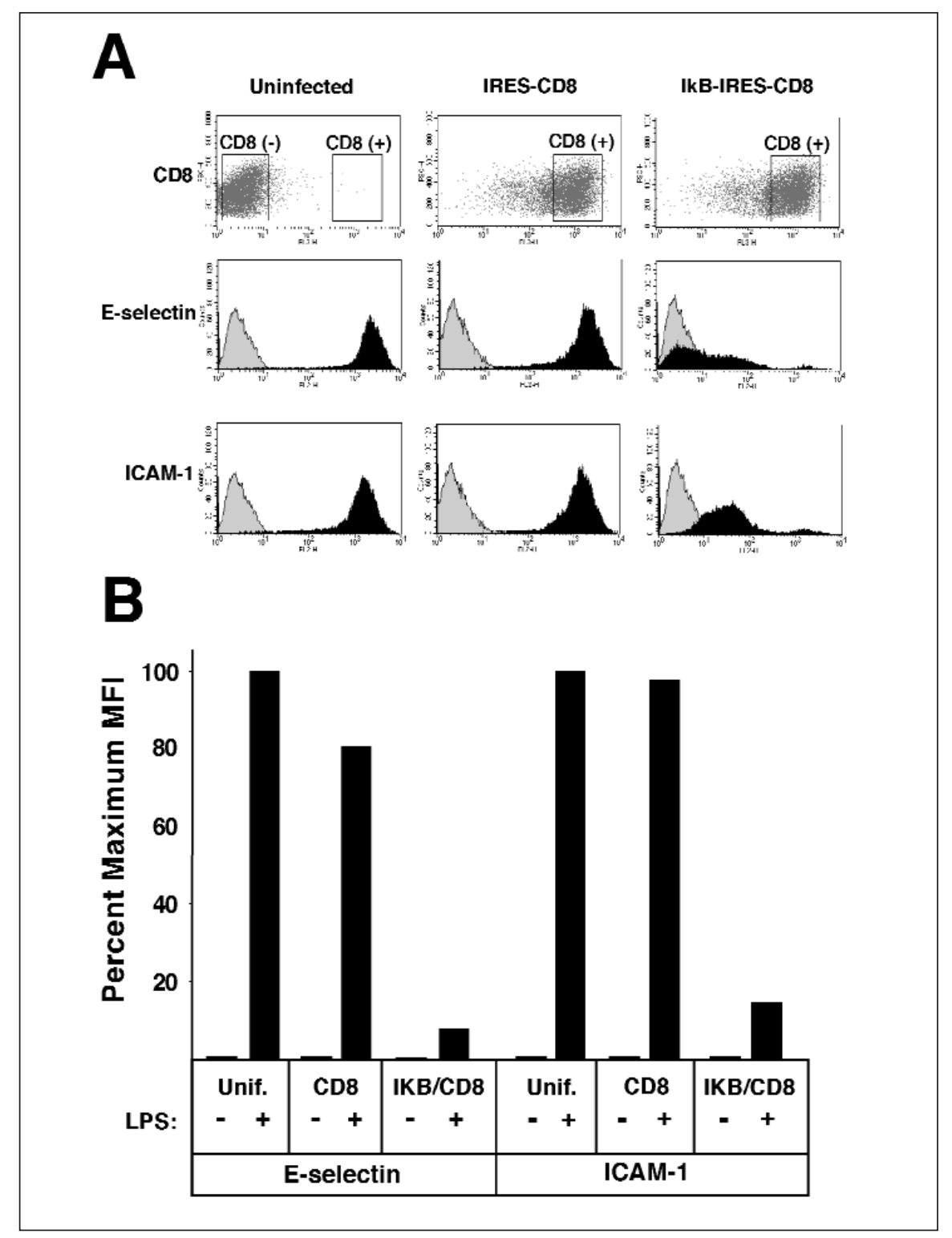

Figure 3. Functional correlation of blockade of endothelial adhesion molecule up-regulation with infection with IKB $\alpha$ mutant-IRES-CD8. (A) HUVECs were infected with control IRES-CD8 or IKB $\alpha$ Ser-IRES-CD8 virus before stimulation with LPS $(500 \mathrm{ng} / \mathrm{mL})$ for $3 \mathrm{~h}$ (E-selectin) or $6 \mathrm{~h}$ (ICAM-1). CD8, E-selectin, and ICAM-1 expression was determined by flow cytometry using PE-conjugated specific antibodies. Uninfected HUVECs were used to define CD8(-) and CD8(+) gates. E-selectin and ICAM-1 expression was then determined for $\mathrm{CD} 8(-)$ uninfected and $\mathrm{CD} 8(+)$ cells infected with the indicated retrovirus. (B) Relative expression of E-selectin and ICAM-1 in CD8(-) uninfected cells and CD8(+) retrovirally infected cells was quantified as percent maximal mean fluorescence intensity (MFI) of the PE (FL2) channel before and after LPS stimulation. Values represent MFI of duplicate samples. 


\section{Research Report}

no need for cell sorting.

An additional advantage of IRESbased expression cassettes is the ability to analyze transduced cells within polyclonal mixtures of infected and uninfected cells. In response to inflammatory stimuli, such as bacterial lipopolysaccharide (LPS), HUVECs rapidly up-regulate the expression of cell surface adhesion molecules, including E-selectin and intracellular adhesion molecule-1 (ICAM-1), which mediate the transmigration of circulating leukocytes into the extravascular space (2). The up-regulation of E-selectin and ICAM-1 is dependent on the activity of the transcription factor nuclear factor$\kappa \mathrm{B}(\mathrm{NF}-\kappa \mathrm{B})$, and inhibition of NF- $\kappa \mathrm{B}$ activation blocks the up-regulation of $\mathrm{E}$ selectin by inflammatory stimuli (1). Additionally, activity of the NF- $\kappa B$ transcription factor can be blocked by overexpression of the endogenous inhibitor of NF- $\kappa B$ (IкB- $\alpha$ ), which contains mutations in two serine residues that prevent its degradation (17). Therefore, we used this system to test the ability of IRES-CD8-based retroviral vectors to functionally define specific subpopulations of cells within polyclonal populations of infected endothelial cells. HUVECs were infected with either control IRES-CD8 or I $\mathrm{I} B \alpha$ Ser ${ }^{32,36}$-IRES-CD8 virus encoding an endogenous NF- $\kappa B$ inhibitor (17). The resulting cell populations were stimulated with LPS for 3 or $6 \mathrm{~h}$ to monitor the expression of E-selectin and ICAM-1, respectively. In response to LPS, uninfected and CD8(-) or CD8(+) cells infected with the control IRES-CD8 virus showed a marked up-regulation of E-selectin and ICAM-1 (Figure 3, A and B). In contrast, $\mathrm{CD} 8(+)$ cells in the population infected with the I $\kappa \mathrm{B} \alpha-\mathrm{Ser}^{32,36}$ IRES-CD8 virus showed a markedly reduced up-regulation of E-selectin and ICAM-1 in response to LPS (Figure 3, $\mathrm{A}$ and $\mathrm{B})$.

The stable integration of retroviral
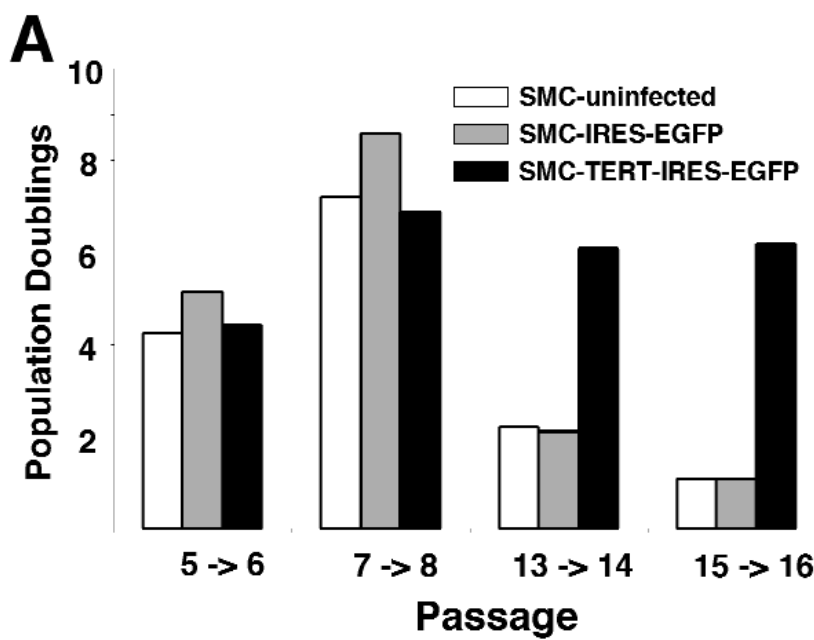

B

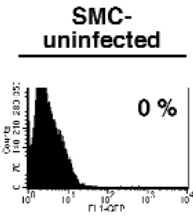

Passage:

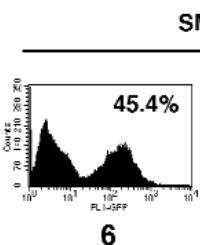

SMC-hTERT-IRES-EGFP

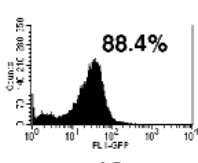

13

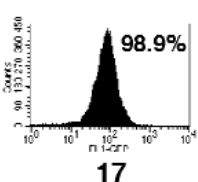

17

Figure 4. In vitro selection of human aortic SMCs infected with hTERT-IRES-EGFP. (A) Human aortic SMCs were infected with control IRES-EGFP or hTERT-IRES-EGFP retrovirus at early passage (P4). At each subsequent passage, $5 \times 10^{5}$ cells were plated and grown for 5-6 days in full growth medium, and total cell number and population doublings per passage were determined for each population. (B) The percentage of EGFP $(+)$ cells in polyclonal mixtures of SMCs infected with hTERT-IRES-EGFP retrovirus was simultaneously determined by flow cytometry as cells grew through normal SMC senescence (P14). 
cassettes into the genomic DNA of target cells provides novel strategies to characterize gene function by monitoring the in vitro evolution of tissue culture populations. Changes in the percentage of $\operatorname{EGFP}(+)$ cells may serve as an indicator of the effects of a co-transduced first cistron gene during positive or negative selective pressures on a population of infected and uninfected cells. For example, expression of a growth inhibitory gene by a retroviral IRES-EGFP cassette will impart a selective growth disadvantage to infected EGFP $(+)$ cells. Over multiple passages in culture, the percentage of $\operatorname{EGFP}(+)$ cells would be predicted to decrease at the expense of the more rapidly growing uninfected EGFP(-) cells. Alternatively, expression of a gene that imparts a selective growth advantage, such as an oncogene, would lead to an increase in the percentage of EGFP $(+)$ cells.

To test this approach in primary vas- cular cells, we characterized the effect of expression of telomerase (hTERT) in human aortic SMCs. Recent studies have shown that expression of hTERT in primary human cells leads to an increased in vitro lifespan through the delay of cellular senescence (18). A cDNA encoding hTERT was cloned into the pBM-IRES-EGFP retroviral vector, and human aortic SMCs were infected with hTERT-IRES-EGFP retrovirus or control IRES-EGFP virus at early passage (Figure 4). At each passage, the percentage of EGFP(+) cells and the doubling rate of the population were determined. Infection of primary SMCs with hTERT-IRES-EGFP virus led to markedly delayed senescence (Figure 4A) and an almost complete enrichment of $\operatorname{EGFP}(+)$ cells as the cells grew through senescence (Figure 4B). Cells infected with control IRES-EGFP virus or uninfected cells underwent senescence after 14 passages or approx- imately 70 population doublings. Additionally, the percentage of $\operatorname{EGFP}(+)$ cells in the control IRES-EGFP-infected population remained constant through senescence (not shown). hTERT-expressing cells maintained a growth rate of 5-6 population doublings per passage for 24 passages and remained approximately $100 \% \operatorname{EGFP}(+)$ for all passages beyond normal senescence (passage 14, not shown).

In this report, we demonstrate that IRES-based retroviral cassettes provide an efficient strategy for the expression of exogenous genes in primary human vascular cells. The excellent correlation between reporter gene expression and cistron 1 gene expression makes it possible to unambiguously identify transduced cells as early as $48 \mathrm{~h}$ after infection. Additionally, gene expression is stable over multiple passages in culture. Reporter gene expression can be readily used to isolate subpopulations 
of cells expressing distinct levels of cistron 1 genes by cell sorting. Retroviral vectors encoding selectable genes, such as the puromycin resistance gene, can also be used to very rapidly generate purified populations of infected primary vascular cells without cell sorting. Alternatively, reporter gene expression can be used to simply identify transduced cells in populations of infected and uninfected cells by flow cytometry or fluorescence microscopy.

Expression strategies, such as those presented, should provide novel approaches for characterizing gene function in primary vascular cells. For example, we have recently shown that retroviral vectors can be used to efficiently deliver NF- $\kappa B$-dependent reporter genes to primary HUVECs to characterize the ability of a co-transduced gene to activate the NF- $\mathrm{\kappa B}$ pathway (11). It should also now be possible to consider expression cloning strategies utilizing IRES-based retroviral cDNA libraries in primary vascular cells, as has recently been reported for other cell types (5). Finally, modifications of existing tetracycline-inducible retroviral expression systems should allow efficient and regulated gene expression in primary vascular cells with a limited in vitro replicative capacity (16).

\section{ACKNOWLEDGMENTS}

We would like to thank Garry Nolan (Stanford University) for generously providing the $\mathrm{pBM}$-series retroviral vectors and Phoenix-A retroviral packaging cells. We gratefully acknowledge the assistance of Kathryn Allen and the Department of Immunology Cell Analysis Facility (University of Washington). This work has been supported by National Institutes of Health grant no. HL18645 (E.W.R.), the Paul G. Allen Foundation for Medical Research (K.J.G.), and a fellowship grant from the Lymphangioleiomyomatosis Foundation (N.F.).

\section{REFERENCES}

1.Boyle, E.M., Jr., J.C. Kovacich, T.G. Canty, Jr., E.N. Morgan, E. Chi, E.D. Verrier, and T.H. Pohlman. 1998. Inhibition of nuclear factor- $\kappa \mathrm{B}$ nuclear localization reduces human E-selectin expression and the systemic inflam- matory response. Circulation 98:II282-II288.

2.Carlos, T.M. and J.M. Harlan. 1994. Leukocyte-endothelial adhesion molecules. Blood 84:2068-2101

3.Gechtman, Z., J.L. Alonso, G. Raab, D.E. Ingber, and M. Klagsbrun. 1999. The shedding of membrane-anchored heparin-binding epidermal-like growth factor is regulated by the Raf/mitogen-activated protein kinase cascade and by cell adhesion and spreading. J. Biol. Chem. 274:28828-28835.

4.Herren, B., E.W. Raines, and R. Ross. 1997. Expression of a disintegrin-like protein in cultured human vascular cells and in vivo. FASEB J. 11:173-180.

5.Hitoshi, Y., J. Lorens, S.I. Kitada, J. Fisher, M. LaBarge, H.Z. Ring, U. Francke, J.C. Reed et al. 1998. Toso, a cell surface, specific regulator of Fas-induced apoptosis in T cells. Immunity 8:461-471.

6.Hobbs, S., S. Jitrapakdee, and J.C. Wallace. 1998. Development of a bicistronic vector driven by the human polypeptide chain elongation factor $1 \alpha$ promoter for creation of stable mammalian cell lines that express very high levels of recombinant proteins. Biochem. Biophys. Res. Commun. 252:368-372.

7.Jang, S.K. and E. Wimmer. 1990. Cap-independent translation of encephalomyocarditis virus RNA: structural elements of the internal ribosomal entry site and involvement of a cellular 57-kD RNA-binding protein. Genes Dev. 4:1560-1572.

8.Kinoshita, S., B.K. Chen, H. Kaneshima, and G.P. Nolan. 1998. Host control of HIV-1 parasitism in $\mathrm{T}$ cells by the nuclear factor of activated T cells. Cell 95:595-604.

9.Kinoshita, S., L. Su, M. Amano, L.A. Timmerman, H. Kaneshima, and G.P. Nolan. 1997. The T cell activation factor NF-ATc positively regulates HIV-1 replication and gene expression in T cells. Immunity 6:235-244.

10.Kovala, A.T., K.A. Harvey, P. McGlynn, G. Boguslawski, J.G. Garcia, and D. English. 2000. High-efficiency transient transfection of endothelial cells for functional analysis. Faseb J. 14:2486-2494

11.Levkau, B., K.J. Garton, N. Ferri, K. Kloke, J.R. Nofer, H.A. Baba, E.W. Raines, and G. Breithardt. 2001. xIAP induces cell-cycle arrest and activates nuclear factor- $\mathrm{\kappa B}$ : new survival pathways disabled by caspase-mediated cleavage during apoptosis of human endothelial cells. Circ. Res. 88:282-290.

12.Mattot, V., C. Vercamer, F. Soncin, T. Calmels, C. Huguet, V. Fafeur, and B. Vandenbunder. 2000. Constitutive expression of the DNA-binding domain of Ets1 increases endothelial cell adhesion and stimulates their organization into capillary-like structures. Oncogene 19:762-772.

13.Morgan, R.A., L. Couture, O. Elroy-Stein, J. Ragheb, B. Moss, and W.F. Anderson. 1992. Retroviral vectors containing putative internal ribosome entry sites: development of a polycistronic gene transfer system and applications to human gene therapy. Nucleic Acids Res. 20:1293-1299.

14.Raab, G. and M. Klagsbrun. 1997. Heparinbinding EGF-like growth factor. Biochim. Biophys. Acta 1333:F179-F199.

15.Ramesh, N., S.T. Kim, M.Q. Wei, M. Kha- lighi, and W.R. Osborne. 1996. High-titer bicistronic retroviral vectors employing footand-mouth disease virus internal ribosome entry site. Nucleic Acids Res. 24:2697-2700.

16.Rossi, F.M., O.M. Guicherit, A. Spicher, A.M. Kringstein, K. Fatyol, B.T. Blakely, and H.M. Blau. 1998. Tetracycline-regulatable factors with distinct dimerization domains allow reversible growth inhibition by $\mathrm{p} 16$. Nat. Genet. 20:389-393.

17.Van Antwerp, D.J., S.J. Martin, I.M. Verma, and D.R. Green. 1998. Inhibition of TNF-induced apoptosis by NF- $\kappa$ B. Trends Cell Biol. 8:107-111.

18.Vaziri, H. and S. Benchimol. 1998. Reconstitution of telomerase activity in normal human cells leads to elongation of telomeres and extended replicative life span. Curr. Biol. 8:279282.

Received 21 June 2001; accepted 19 November 2001.

Address correspondence to:

Dr. Elaine W. Raines

Department of Pathology

Harborview Medical Center

325 9th Avenue, Box 359675

Seattle, WA 98104-2499, USA

e-mail:ewraines@u.washington.edu 